(C) 2016 IEEE. Personal use of this material is permitted. Permission from IEEE must be obtained for all other uses, in any current or future media, including reprinting/republishing this material for advertising or promotional purposes, creating new collective works, for resale or redistribution to servers or lists, or reuse of any copyrighted component of this work in other works. 


\title{
Localization Algorithm Design and Performance Analysis in Probabilistic LOS/NLOS Environment
}

\author{
Xiufang Shi*, Guoqiang Mao ${ }^{\ddagger}, \dagger, \S$, Zaiyue Yang* and Jiming Chen* \\ ${ }^{*}$ State Key Lab. of Industrial Control Technology, Zhejiang University, China \\ ${ }^{\ddagger}$ School of Computing and Communication, University of Technology Sydney, Australia \\ ${ }^{\dagger}$ Data61 Australia \\ ${ }^{\S}$ School of Information and Communication Engineering, Beijing University of Posts and Telecommunications, China \\ Email:xfshi.zju@gmail.com, guoqiang.mao@uts.edu.au,yangzy@zju.edu.cn, jmchen@ieee.org
}

\begin{abstract}
Non-line-of-sight (NLOS) propagation, which widely exists in wireless systems, will degrade the performance of wireless positioning system if it is not taken into consideration in the localization algorithm design. The 3rd Generation Partnership Project (3GPP) suggests that the probabilities of lineof-sight (LOS) and NLOS are related to the distance between the receiver and the transmitter. In this paper, we propose a Maximum Likelihood Estimator (MLE) for localization, which incorporates the distance dependent LOS/NLOS probabilities. Then, the position error bound is derived using Cramer-Rao Lower Bound (CRLB). Through numerical analysis, the impact of NLOS propagation on the position error bound is evaluated. The performance of our proposed algorithm is verified by real world experimental data.
\end{abstract}

\section{INTRODUCTION}

Wireless technologies promote the great development of a large amount of applications [1]-[7]. Driven by the increasing demand from location-based services, wireless localization systems have attracted significant research interest in recent years. Numerous localization systems have been developed, e.g. Global Positioning System (GPS), Cellularbased Positioning System (CPS), Wireless Local Area Network (WLAN)-based positioning system, etc. These positioning systems can deliver very good performance in ideal environments, i.e., line-of-sight (LOS) propagated environments. In practical environment, especially, indoors or urban areas, wireless signals often suffer from non-line-of-sight (NLOS) propagation, which will significantly degrade the localization performance of the conventional localization techniques under the LOS assumptions.

Many techniques have been developed to deal with localization problem in NLOS conditions [8]-[13]. It can be roughly divided into two categories: a) identify and discardbased approach [8]-[10]; b) weighted combination of LOS and NLOS measurements [12], [13]. In the first approach, the LOS/NLOS paths are first identified using statistical techniques. By analyzing the channel features, e.g., mean and standard deviation [8], Kurtosis [10], Rician K factor [10], etc., the NLOS paths can be identified by hypothesis testing. Then, the identified NLOS measurements are discarded. Only the LOS measurements are utilized for localization. However, this kind of technique needs sufficient number of LOS measurements, which renders it not suitable for environments with only a small number of base stations. The second approach does not need to identify the NLOS measurements in advance.
All the measurements are used for localization. By taking residual error [12], noise variance [13], etc., as the weights, the position estimate will be obtained through Least Square (LS) or Maximum Likelihood Estimator (MLE). This approach is widely used in environments with a small number of base stations.

Generally, when the distance between a receiver and a transmitter is small, LOS propagations are more likely to occur, and when the distance between the receiver and the transmitter increases, the probability of LOS will decrease, and the signals will suffer from NLOS propagation with a higher probability. This phenomenon is also verified by the $3 \mathrm{rd}$ Generation Partnership Project (3GPP) [6], [7]. Even though, there are a number of existing algorithms considering the LOS/NLOS probabilities [9], [14], they usually assume the LOS/NLOS probabilities are fixed and known a priori, which does not capture the fact that the occurrence of LOS and NLOS propagations varies with the distance between the transmitter and the receiver, or the distance dependent LOS/NLOS probabilities are inappropriately modeled [15]. To the best of our knowledge, there is still no work that takes the distance dependent LOS/NLOS probabilities as mentioned in 3GPP [6], [7] into localization algorithm design and performance analysis.

In this paper, we will study the localization problem considering distance dependent LOS/NLOS probabilities. The main contributions of this paper are summarized as follows:

- We propose an MLE-based estimation algorithm for a single target localization problem, which incorporates the distance dependent LOS/NLOS probabilities.

- Cramer-Rao Lower Bound (CRLB) is used to analyze the localization accuracy considering a general NLOS bias model. Localization performances under four different and widely used distributions of NLOS bias are numerically analyzed.

- The performance of the proposed localization algorithm is verified in a realistic scenario using range measurements obtained by ultra-wide bandwidth (UWB) devices.

The remainder of this paper is organized as follows. Section II introduces the system model. Section III presents the MLE-based localization algorithm. CRLB-based localization accuracy analysis is conducted in section IV. Experimental results are presented in section $\mathrm{V}$. Conclusions are drawn in 
section VI.

\section{SySTEM MODEL}

We consider a 2-D positioning system with $N$ base stations (BSs) whose positions are known as $X_{i}=\left[x_{i}, y_{i}\right]^{T}, i=$ $1, \cdots, N$. The position of the mobile station (MS), i.e. the target, denoted as $X=[x, y]^{T}$, is unknown and needs to be estimated. It is assumed that the noisy range measurements between the MS and the BSs can be obtained, which are expressed as

$$
z_{i}=d_{i}+\varepsilon_{i}, \quad i=1, \cdots, N
$$

where $d_{i}=\sqrt{\left(x_{i}-x\right)^{2}+\left(y_{i}-y\right)^{2}}$ is the true distance between the MS and the $i$-th BS and $\varepsilon_{i}$ is measurement error. The measurement error is usually modeled as zeromean white Gaussian noise under LOS propagation [13], [16], [17]. In practice, because of the sophisticated propagation environment, not all the paths are LOS paths. If path $i$ is an NLOS path, $\varepsilon_{i}$ also includes an NLOS bias in addition to the additive Gaussian noise. Consequently, the measurement error can be modeled as follows

$$
\varepsilon_{i}=\left\{\begin{array}{cc}
v_{i}, & \text { LOS path } \\
b_{i}+v_{i}, & \text { NLOS path }
\end{array}\right.
$$

where $v_{i} \sim \mathcal{N}\left(0, \sigma_{i}^{2}\right)$ is the zero-mean white Gaussian noise, and $b_{i}$ is the bias attributable to NLOS propagation. In the literature the bias term has been modeled as Gaussian distribution [13], exponential distribution [16], uniform distribution [17], or just being a constant [13]. In this paper, we assume the distributions of $v_{i}$ and $b_{i}$ are known a priori, which can be obtained from field measurements. The measurement errors in different paths are assumed to be independent. Moreover, $v_{i}$ and $b_{i}$ in the same NLOS path are also assumed to be independent [13], [16], [17]. Consequently, the probability density function (PDF) of the measurement error $\varepsilon_{i}$ under LOS propagation is the PDF of the Gaussian variable $v_{i}$,

$$
p^{L}\left(\varepsilon_{i}\right)=\frac{1}{\sqrt{2 \pi \sigma_{i}^{2}}} \exp \left(-\frac{\varepsilon_{i}^{2}}{2 \sigma_{i}^{2}}\right)
$$

The PDF of $\varepsilon_{i}$ under NLOS propagation is the PDF of the sum of two independent random variables, i.e., $v_{i}+b_{i}$, which can be derived through convolution

$$
p^{N L}\left(\varepsilon_{i}\right)=\int_{-\infty}^{\infty} p^{L}\left(\varepsilon_{i}-\tau\right) p_{b_{i}}(\tau) d \tau
$$

where $p_{b_{i}}$ stands for the PDF of $b_{i}$. The analytical form of $p^{N L}\left(\varepsilon_{i}\right)$ with $b_{i}$ assuming four different distributions as mentioned previously can be derived as follows:

- Gaussian distributed NLOS bias: $b_{i} \sim \mathcal{N}\left(\mu_{b i}, \sigma_{b i}^{2}\right)$ :

$$
p_{G}^{N L}\left(\varepsilon_{i}\right)=\frac{1}{\sqrt{2 \pi \sigma_{\varepsilon i}^{2}}} \exp \left(-\frac{\left(\varepsilon_{i}-\mu_{\varepsilon i}\right)^{2}}{2 \sigma_{\varepsilon i}^{2}}\right)
$$

where $\mu_{\varepsilon i}=\mu_{b i}$ and $\sigma_{\varepsilon i}^{2}=\sigma_{b i}^{2}+\sigma_{i}^{2}$.

- Exponentially distributed NLOS bias: $b_{i} \sim \operatorname{Exp}(\lambda)$, where $\lambda>0$ is the parameter of the distribution:

$$
p_{E}^{N L}\left(\varepsilon_{i}\right)=\frac{\lambda}{2} \exp \left(-\lambda\left(\varepsilon_{i}-\frac{\lambda \sigma_{i}^{2}}{2}\right)\right) \operatorname{erfc}\left(\frac{\lambda \sigma_{i}^{2}-\varepsilon_{i}}{\sqrt{2} \sigma_{i}}\right)
$$

where $\operatorname{erfc}(\cdot)$ denotes the complementary error function.

- Uniformly distributed NLOS bias: $b_{i} \sim U\left(l b_{i}, u b_{i}\right)$, where $l b_{i}$ and $u b_{i}$ are the lower bound and upper bound of this uniform distribution, respectively:

$$
p_{U}^{N L}\left(\varepsilon_{i}\right)=\frac{1}{\left(u b_{i}-l b_{i}\right)}\left(\Phi\left(\frac{u b_{i}-\varepsilon_{i}}{\sigma_{i}}\right)-\Phi\left(\frac{l b_{i}-\varepsilon_{i}}{\sigma_{i}}\right)\right)
$$

where $\Phi(\cdot)$ denotes the cumulative distribution function (CDF) of the standard normal distribution.

- Constant NLOS bias: $b_{i}=b$ :

$$
p_{C}^{N L}\left(\varepsilon_{i}\right)=\frac{1}{\sqrt{2 \pi \sigma_{i}^{2}}} \exp \left(-\frac{\left(\varepsilon_{i}-b\right)^{2}}{2 \sigma_{i}^{2}}\right)
$$

According to 3GPP [6], the occurrence of LOS path depends on the MS-BS distance $d_{i}$, whose probability is denoted as $p\left(L O S \mid d_{i}\right)$ and NLOS probability is $p\left(N L O S \mid d_{i}\right)=$ $1-p\left(L O S \mid d_{i}\right)$. The LOS probability is modeled as different forms under different environmental conditions, including indoor hotspots, urban micro, urban macro and rural macro cellular networks [6]. For example, as shown in [6], the LOS probability in indoor environment is

$$
p\left(L O S \mid d_{i}\right)=\left\{\begin{array}{cc}
1, & 0<d_{i} \leq 18 \\
\exp \left(-\frac{d_{i}-18}{27}\right), & 18<d_{i}<37 \\
0.5, & d_{i} \geq 37
\end{array}\right.
$$

We can see with the increase of $d_{i}$, the LOS probability will decrease. It has been shown that it is important to take into account the distance dependent LOS/NLOS probabilities in system design and performance analysis [4], [18].

In this paper, we propose a localization algorithm to estimate the position of the MS, which 1) considers the probabilities of LOS and NLOS; 2) uses both LOS and NLOS measurements; 3) does not require identification of LOS and NLOS paths before localization. As mentioned before, the priori identification of LOS and NLOS paths can be difficult when the number of measurements is small. Then we analyze the localization performance with NLOS biases assuming four different distributions mentioned previously. Both numerical and experimental studies demonstrate that it is important to consider both LOS and NLOS paths in localization, which results in better localization accuracy.

\section{MLE-BASED LOCALIZATION}

In this work, we assume the distributions of measurement noise and NLOS bias are known, along with the distance dependent LOS/NLOS probabilities, which makes MLE the preferred option in estimating the MS's position, since MLE is maximizing the joint likelihood function of MS's position.

Incorporating the LOS and NLOS probabilities, the PDF of measurement $z_{i}$ is expressed as

$p_{z}\left(z_{i} \mid X\right)=p_{z}^{L}\left(z_{i} \mid X\right) p\left(L O S \mid d_{i}\right)+p_{z}^{N L}\left(z_{i} \mid X\right) p\left(N L O S \mid d_{i}\right)$

where $p_{z}^{L}\left(z_{i} \mid X\right)$ is the PDF of $z_{i}$ under LOS propagation, which can be easily obtained from (3) as

$$
p_{z}^{L}\left(z_{i} \mid X\right)=p^{L}\left(z_{i}-d_{i}\right)
$$

and $p_{z}^{N L}\left(z_{i} \mid X\right)$ is the PDF of $z_{i}$ under NLOS propagation, 
which can be obtained from (4) as

$$
p_{z}^{N L}\left(z_{i} \mid X\right)=p^{N L}\left(z_{i}-d_{i}\right)
$$

The expression $p^{N L}\left(z_{i}-d_{i}\right)$ varies with different distributions of NLOS bias, as discussed in Section II. Since the measurement errors in different paths are mutual independent, the joint PDF of the whole positioning system is

$$
P(Z \mid X)=\prod_{i=1}^{N} p_{z}\left(z_{i} \mid X\right)
$$

where $Z=\left[z_{1}, \cdots, z_{N}\right]^{T}$ is the measurement vector. The estimate of $X$ by MLE can be obtained as

$$
\hat{X}=\arg \max _{X} \ln P(Z \mid X)=\arg \max _{X} \sum_{i=1}^{N} \ln p_{z}\left(z_{i} \mid X\right)
$$

This MLE-based algorithm does not need to identify the NLOS paths beforehand and takes full advantage of all the available measurements, including both the LOS measurements and the NLOS measurements. It will achieve the optimum result assuming that the distribution of measurement error is known. The solution can usually be obtained by Gauss-Newton method starting from a good initial estimation.

\section{CRLB-BASED PERformance AnAlysis}

In this section, we will analyze the fundamental limits of localization accuracy in probabilistic LOS/NLOS environment using CRLB, which is often utilized as a benchmark to evaluate the estimation accuracy [13], [16], [19], [20].

\section{A. Position Error Bound}

For a localization problem in $\mathbb{R}^{2}$, the CRLB is a $2 \times 2$ matrix indicating the lower bound of the covariance matrix of estimation error. And the position error bound (PEB) is usually taken as a scalar metric for localization accuracy, which is defined as

$$
\operatorname{PEB}(X)=\sqrt{\operatorname{Tr}(C R L B(X))}=\sqrt{\operatorname{Tr}\left(J(X)^{-1}\right)}
$$

where $\operatorname{Tr}(\cdot)$ denotes the trace operation of one matrix and $J(X)$ is the Fisher Information Matrix (FIM) at $X$, which is defined as

$$
J(X)=\mathbb{E}\left[\begin{array}{cc}
\left(\frac{\partial \ln P(Z \mid X)}{\partial x}\right)^{2} & \frac{\partial \ln P(Z \mid X)}{\partial x} \frac{\partial \ln P(Z \mid X)}{\partial y} \\
\frac{\partial \ln P(Z \mid X)}{\partial y} \frac{\partial \ln P(Z \mid X)}{\partial x} & \left(\frac{\partial \ln P(Z \mid X)}{\partial y}\right)^{2}
\end{array}\right]
$$

The entry of $J(X)$ at the fist row and the first column is computed as follows

$$
\begin{aligned}
J_{11}(X)= & \mathbb{E}\left[\left(\sum_{i=1}^{N} \frac{\partial \ln p_{z}\left(z_{i} \mid X\right)}{\partial x}\right)^{2}\right] \\
= & \mathbb{E}\left[\sum_{i=1}^{N}\left(\frac{\partial \ln p_{z}\left(z_{i} \mid X\right)}{\partial x}\right)^{2}\right] \\
& +2 \mathbb{E}\left[\sum_{i=1}^{N} \sum_{j>i}^{N} \frac{\partial \ln p_{z}\left(z_{i} \mid X\right)}{\partial x} \frac{\partial \ln p_{z}\left(z_{j} \mid X\right)}{\partial x}\right] \\
= & f_{1}(X)+2 f_{2}(X)
\end{aligned}
$$

where

$$
\begin{aligned}
f_{1}(X) & =\sum_{i=1}^{N} \mathbb{E}\left[\left(\frac{\partial \ln p_{z}\left(z_{i} \mid X\right)}{\partial x}\right)^{2}\right] \\
& =\sum_{i=1}^{N} \mathbb{E}\left[\left(\frac{\partial \ln p_{z}\left(z_{i} \mid X\right)}{\partial d_{i}}\right)^{2}\left(\frac{\partial d_{i}}{\partial x}\right)^{2}\right]
\end{aligned}
$$

and

$$
\begin{aligned}
& f_{2}(X) \\
& =\mathbb{E}\left[\sum_{i=1}^{N} \sum_{j>i}^{N} \frac{\partial \ln p_{z}\left(z_{i} \mid X\right)}{\partial x} \frac{\partial \ln p_{z}\left(z_{j} \mid X\right)}{\partial x}\right] \\
& =\sum_{i=1}^{N} \sum_{j>i}^{N} \mathbb{E}\left[\frac{\partial \ln p_{z}\left(z_{i} \mid X\right)}{\partial d_{i}} \frac{\partial \ln p_{z}\left(z_{j} \mid X\right)}{\partial d_{j}}\right] \frac{\partial d_{i}}{\partial x} \frac{\partial d_{j}}{\partial x}
\end{aligned}
$$

Let $\cos \theta_{i}=\frac{\partial d_{i}}{\partial x}=\frac{x-x_{i}}{d_{i}}$ and $\sin \theta_{i}=\frac{\partial d_{i}}{\partial y}=\frac{y-y_{i}}{d_{i}}$, then $f_{1}(X)$ can be rewritten as

$$
f_{1}(X)=\sum_{i=1}^{N} \mathbb{E}\left[\left(\frac{\partial \ln p_{z}\left(z_{i} \mid X\right)}{\partial d_{i}}\right)^{2}\right] \cos ^{2} \theta_{i}
$$

Since the measurement errors in different paths are independent, it follows that

$$
\begin{aligned}
& \mathbb{E}\left[\frac{\partial \ln p_{z}\left(z_{i} \mid X\right)}{\partial d_{i}} \frac{\partial \ln p_{z}\left(z_{j} \mid X\right)}{\partial d_{j}}\right] \\
& =\mathbb{E}\left[\frac{\partial \ln p_{z}\left(z_{i} \mid X\right)}{\partial d_{i}}\right] \mathbb{E}\left[\frac{\partial \ln p_{z}\left(z_{j} \mid X\right)}{\partial d_{j}}\right]
\end{aligned}
$$

Moreover, from the regulation condition of CRLB in [20],

$$
\mathbb{E}\left[\frac{\partial \ln p_{z}\left(z_{i} \mid X\right)}{\partial d_{i}}\right]=0
$$

Thus, $f_{2}(X)=0$. Consequently,

$$
J_{11}(X)=f_{1}(X)=\sum_{i=1}^{N} \mathbb{E}\left[\left(\frac{\partial \ln p_{z}\left(z_{i} \mid X\right)}{\partial d_{i}}\right)^{2}\right] \cos ^{2} \theta_{i}
$$

Accordingly, the other entries of $J(X)$ are obtained as

$$
\begin{gathered}
J_{12}(X)=J_{21}(X)= \\
\sum_{i=1}^{N} \mathbb{E}\left[\left(\frac{\partial \ln p_{z}\left(z_{i} \mid X\right)}{\partial d_{i}}\right)^{2}\right] \cos \theta_{i} \sin \theta_{i} \\
J_{22}(X)=\sum_{i=1}^{N} \mathbb{E}\left[\left(\frac{\partial \ln p_{z}\left(z_{i} \mid X\right)}{\partial d_{i}}\right)^{2}\right] \sin ^{2} \theta_{i}
\end{gathered}
$$

Let $f\left(d_{i}\right)=\mathbb{E}\left[\left(\frac{\partial \ln p_{z}\left(z_{i} \mid X\right)}{\partial d_{i}}\right)^{2}\right]$, then $J(X)$ is expressed as

$$
J(X)=\sum_{i=1}^{N} f\left(d_{i}\right)\left[\begin{array}{cc}
\cos ^{2} \theta_{i} & \cos \theta_{i} \sin \theta_{i} \\
\cos \theta_{i} \sin \theta_{i} & \sin ^{2} \theta_{i}
\end{array}\right]
$$

\section{B. Numerical Analysis}

From (26) and (15), we can find that, the value of $f\left(d_{i}\right)$ will affect the value of PEB. A larger $f\left(d_{i}\right)$ implies a lower PEB. The NLOS bias and LOS/NLOS probabilities can only 
affect the value of $f\left(d_{i}\right)$, therefore, we just need to analyze the influence of NLOS propagation on $f\left(d_{i}\right)$. And $f\left(d_{i}\right)$ can be further computed as

$$
f\left(d_{i}\right)=\mathbb{E}\left[\left(\frac{\partial \ln p_{z}\left(z_{i} \mid X\right)}{\partial d_{i}}\right)^{2}\right]=\int_{-\infty}^{+\infty} \frac{\left(\frac{\partial p_{z}\left(z_{i} \mid X\right)}{\partial d_{i}}\right)^{2}}{p_{z}\left(z_{i} \mid X\right)} d z_{i}
$$

Since we incorporate the LOS/NLOS probabilities and NLOS bias into $p_{z}\left(z_{i} \mid X\right)$, as shown in (10), consequently, the analytical form of $p_{z}\left(z_{i} \mid X\right)$ is complicated, whose partial derivative over $d_{i}$ is much more complicated. As a result, the integral in (27) is difficult to express analytically. We resort to using a numerical method, i.e., Gauss-Hermite quadrature, to approximate the integral in (27). Let $K$ be the number of sample points used in Gauss-Hermite quadrature, $t_{k}, k=1, \cdots, K$ be the zero points of the physicists' version of the Hermite polynomial, whose associated weight is $\omega_{k}$. The values of zero points and weights of Hermite polynomial can be found in the table shown in [21]. Then $f\left(d_{i}\right)$ can be approximated as follows

$$
f\left(d_{i}\right) \approx \sum_{k=1}^{K} \omega_{k} h\left(t_{k}\right)
$$

where

$$
h\left(t_{k}\right)=e^{t_{k}^{2}} \frac{\left(\frac{\partial p_{z}\left(t_{k} \mid X\right)}{\partial d_{i}}\right)^{2}}{p_{z}\left(t_{k} \mid X\right)}
$$

The approximation error

$$
R_{K}=f\left(d_{i}\right)-\sum_{k=1}^{K} \omega_{k} h\left(t_{k}\right)=\frac{K ! \sqrt{\pi}}{2^{K}(2 K) !} h^{(2 K)}(\xi)
$$

where $h^{(2 K)}(\xi)$ denotes the $2 K$ order derivative of $h$ at some $\xi$ in $(-\infty, \infty)$ [22].

To investigate the influence of LOS/NLOS probabilities and NLOS biases on the localization performance, in the numerical analysis, we adopt the LOS probability model in (9) for indoor environment [6]. The parameters in the measurement error model are set to be the same as those in [13], i.e., the additive Gaussian noise $v_{i} \sim \mathcal{N}(0,1)$, the mean and standard deviation of NLOS bias are respectively $\mu=2$ and $\sigma=2$. The function of $f\left(d_{i}\right)$ is computed as introduced before with $b_{i}$ assuming Gaussian distribution $b_{i} \sim \mathcal{N}\left(\mu, \sigma^{2}\right)$, uniform distribution $b_{i} \sim U(\mu-\sqrt{3} \sigma, \mu+\sqrt{3} \sigma)$, exponential distribution $b_{i} \sim \operatorname{Exp}(1 / \mu)$ and constant $b_{i}=\mu$. The number of Gauss-Hermite sample points is chosen to be $K=100$.

Fig. 1 shows the impact of NLOS bias on $f\left(d_{i}\right)$. In this figure, when $d_{i} \leq 18$, no NLOS propagation occurs. When $18<d_{i}<37$, with the increase of $d_{i}, p\left(N L O S \mid d_{i}\right)$ increases, the impact of NLOS bias on $f\left(d_{i}\right)$ increases. When $d_{i} \geq 37$, $p\left(N L O S \mid d_{i}\right)$ keeps unchanged, the impact of NLOS bias on $f\left(d_{i}\right)$ becomes stable. We can see NLOS biases with different distributions have different impacts on $f\left(d_{i}\right)$. Since a lower $f\left(d_{i}\right)$ indicates a higher PEB, we can say that the PEB associated with a Gaussian distributed bias is larger than the PEB with a uniformly distributed bias and again larger than the PEB with an exponentially distributed bias. Constant bias results in the smallest PEB.

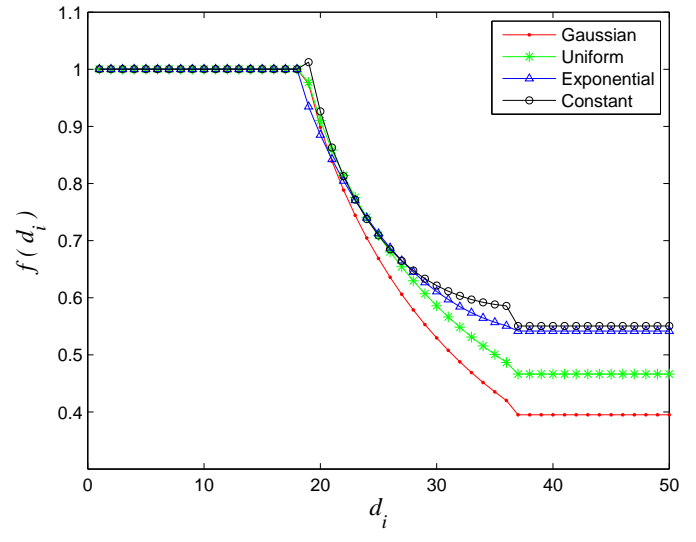

Fig. 1. $f\left(d_{i}\right)$ with NLOS biases under four different distributions with the common mean and standard deviation, i.e., $\mu=2, \sigma=2$

\section{EXPERIMENTAL RESULTS}

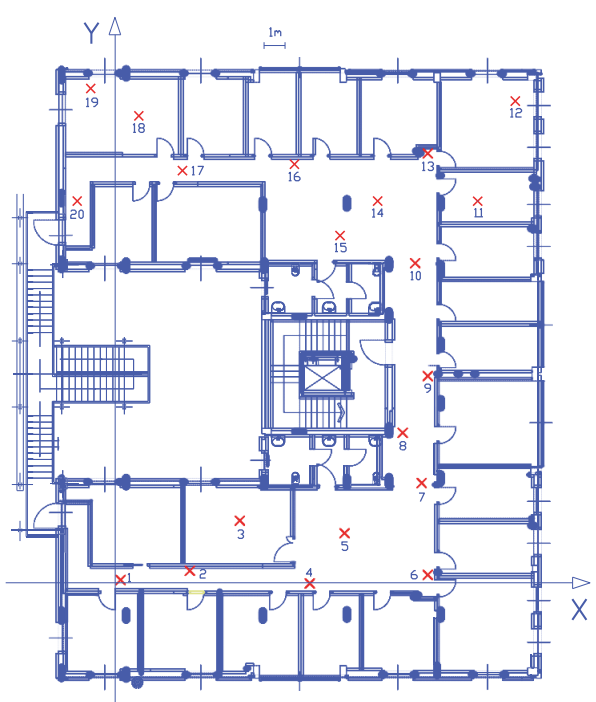

Fig. 2. The experimental environment in WiLab, University of Bologna, Cesena Campus [23]

In this section, we test the performance of the proposed localization algorithm using the measurements database WPR.B in [23]. This database includes extensive range measurements which are taken by 2 commercial UWB devices, PulsON220, in a typical indoor office environment, as shown in Fig. 2. In Fig. 2, a grid of 20 static positions, marked by red crosses, are used for the UWB measurements. By putting the UWB devices at two of the 20 positions, the time of arrival (TOA) ranging measurements between these two points can be obtained and stored as a range measurement vector, which includes more than 200 range measurements. Because of the strong attenuations and limited radiated power of the UWB devices, not all UWB devices in the 20 static positions can communicate with each other. There exist only 69 links where the UWB devices can communicate with each other and conduct ranging. In these 69 links, some of them are LOS, and the others are NLOS. We will first derive the model of LOS probability using this database. Then, we will localize the target at these 20 positions using the corresponding range measurements and the LOS/NLOS probabilities. 
Ideally, we would want to use measurements obtained from cellular networks to validate the performance of the proposed localization algorithm. Limited by experimental conditions, here we use indoor UWB measurements instead. However this does not affect the main focus of this paper, i.e. it is important to consider both LOS and NLOS propagations in localization.

1) LOS probability fitting: About the MS-BS distance related LOS probability model, we mainly consider two models:

a) Linear model proposed in [7]:

$$
p_{l}(L O S \mid d)=\alpha_{l} d+\beta_{l}
$$

where $\alpha_{l}$ and $\beta_{l}$ are the linear model parameters;

b) Exponential model proposed in [6]:

$$
p_{e}(L O S \mid d)=\exp \left(\frac{-\left(d-\alpha_{e}\right)}{\beta_{e}}\right)
$$

where $\alpha_{e}$ and $\beta_{e}$ are the exponential model parameters. In our experimental environment, the LOS probability is always changing with the distance, that is why we dismiss the parts where the LOS probability keeps unchanged in (9).

In the database, there are 33 LOS links with true internode distances $D_{\text {los }}=\left[d_{1}^{\text {los }}, \cdots, d_{33}^{\text {los }}\right]$, and 36 NLOS links with true internode distances $D_{\text {nlos }}=\left[d_{1}^{\text {nlos }}, \cdots, d_{36}^{\text {nlos }}\right]$. We use MLE to estimate the LOS probability model parameters in (31) and (32). The PDF of $\alpha_{l}$ and $\beta_{l}$ is computed as

$$
\begin{aligned}
& P\left(\alpha_{l}, \beta_{l}\right)=\prod_{i=1}^{33} p_{l}\left(\operatorname{LOS} \mid d_{i}^{\text {los }}\right) \prod_{j=1}^{36} p_{l}\left(N L O S \mid d_{j}^{\text {nlos }}\right) \\
& =\prod_{i=1}^{33}\left(\alpha_{l} d_{i}^{\text {los }}+\beta_{l}\right) \prod_{j=1}^{36}\left(1-\left(\alpha_{l} d_{j}^{\text {nlos }}+\beta_{l}\right)\right)
\end{aligned}
$$

Correspondingly, the PDF of $\alpha_{e}$ and $\beta_{e}$ is computed as

$$
\begin{aligned}
& P\left(\alpha_{e}, \beta_{e}\right)=\prod_{i=1}^{33} p_{e}\left(L O S \mid d_{i}^{l o s}\right) \prod_{j=1}^{36} p_{e}\left(N L O S \mid d_{j}^{\text {nlos }}\right) \\
& =\prod_{i=1}^{33} \exp \left(\frac{-\left(d_{i}^{l o s}-\alpha_{e}\right)}{\beta_{e}}\right) \prod_{j=1}^{36}\left(1-\exp \left(\frac{-\left(d_{j}^{\text {nlos }}-\alpha_{e}\right)}{\beta_{e}}\right)\right)
\end{aligned}
$$

The estimates of $\alpha_{l}, \beta_{l}, \alpha_{e}$ and $\beta_{e}$ are

$$
\begin{gathered}
\left(\hat{\alpha_{l}}, \hat{\beta}_{l}\right)=\arg \max _{\alpha_{l}, \beta_{l}} \ln P\left(\alpha_{l}, \beta_{l}\right) \\
\left(\hat{\alpha_{e}}, \hat{\beta}_{e}\right)=\arg \max _{\alpha_{e}, \beta_{e}} \ln P\left(\alpha_{e}, \beta_{e}\right)
\end{gathered}
$$

The estimated LOS probability models for this database are

$$
\begin{gathered}
p_{l}(L O S \mid d)=-0.018 d+0.6237 \\
p_{e}(L O S \mid d)=\exp \left(\frac{-(d+4.7901)}{16.6700}\right)
\end{gathered}
$$

The fitted results under different probability models are shown in Fig.3. The Mean Square Errors (MSE) of the fitted models (37) and (38) are almost equal. Considering that the linear model is simpler than the exponential model to analyze, we would apply the linear probability model (37) to the localization.

2) Localization results: In the localization part, we use the range measurements provided in the WPR.B database.

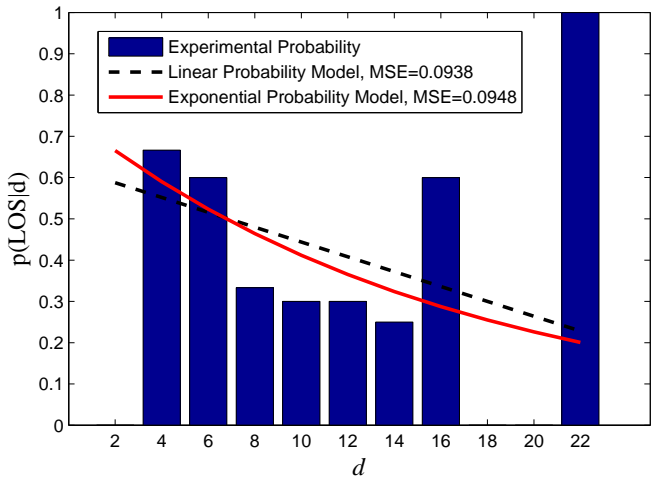

Fig. 3. Fitted results of LOS probability.

Through numerical fitting using the range measurements in the whole database, the range measurement error under LOS condition follows a Gaussian distribution, i.e. $\varepsilon_{L} \sim$ $\mathcal{N}(-0.0214,0.1149)$, and the range measurement error under NLOS condition also follows a Gaussian distribution, i.e. $\varepsilon_{N L} \sim \mathcal{N}(0.7613,0.9860)$. The NLOS bias is found to be Gaussian distributed. In the localization process, we assume the target is at one of the 20 static positions and the neighbors of this target are anchors, whose positions are assumed to be known. Then we use range measurements between this target and its neighbors to estimate the target's position by the MLE proposed in section III combining the LOS probability model in (37). For example, if we want to localize the target at position 1 , the anchors for the target are $2,3,4,6,7$, the range measurements between 1 and 2, 3, 4, 6, 7 are utilized for localization. Fig. 4 shows the localization results for all the 20 points using only one range measurement for each pair of neighbors. In this figure, MLE-PC represents the location estimates obtained by our proposed algorithm; MLE$\mathrm{AL}$ represents the location estimates obtained by the MLE estimator assuming all the paths are LOS; MLE-TC represents the location estimates obtained by the MLE estimator with accurate knowledge of LOS and NLOS paths. From the illustration of this figure, and the Root Mean Square Errors (RMSEs) of these three methods, we can see the performance of our proposed MLE-PC is the best, compared with MLE-AL and MLE-TC. Moreover, we conduct this localization for 200 times, i.e., using 200 different range measurements for each pair of neighbors. Fig.5 illustrates the CDF of the position estimation error in 200 runs, which further shows that our proposed MLE-PC outperforms MLE-AL and MLE-TC.

At the first sight, it might be confusing that MLE-PC is better than MLE-TC. The reason behind this phenomenon is the statistics of LOS and NLOS are obtained by fitting technique, which incorporates all the measurements at the 20 positions. There exist many outliers in this database that may not fit well the obtained statistical model. For example, at position number 11, all the measurements are under NLOS condition, despite that the target at position 11 has small distances to its neighbors, whereas the actual errors in the range measurements obtained at position 11 are all very small, which make the associated measurements more like LOS measurements. In our MLE-PC algorithm, since the distances between 11 and its 
neighbors are small, those range measurements are taken as LOS measurements with a higher probability. And in MLE$\mathrm{TC}$, all the measurements are taken as NLOS measurements (which are the true conditions) with larger mean and larger standard deviation. As a result, the location estimates obtained by MLE-TC which has accurate knowledge of LOS and NLOS paths are worse than that obtained by MLE-PC.

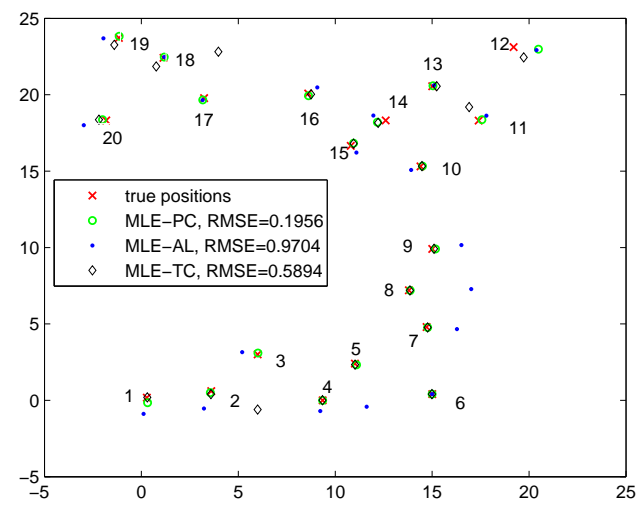

Fig. 4. Localization results in a single run.

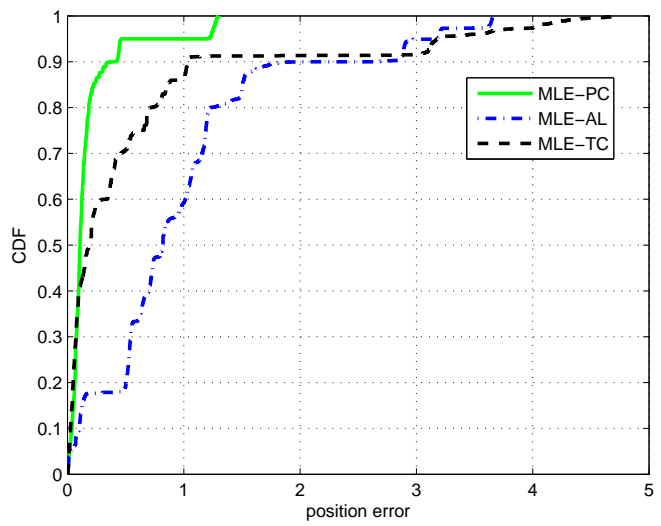

Fig. 5. CDF of position estimation errors of 200 runs.

\section{CONCLUSION}

In this paper, the localization problem in an environment including both LOS and NLOS paths is investigated. By combining the distance dependent LOS/NLOS probabilities, an MLE-based localization algorithm is proposed. The PEB for general LOS/NLOS probability model and NLOS bias model is derived. The numerical analysis shows that Gaussian NLOS bias leads to the worst localization performance, and constant NLOS bias leads to the best localization performance. Through experiments using real UWB measurements, we show that the performance of our proposed algorithm is better than that of MLE with all measurements assumed to be LOS, and it is also better than that of MLE with the true LOS/NLOS conditions.

\section{ACKNOWLEDGMENT}

Zaiyue Yang's work is supported by 973 Program under Grant 2013CB329503, NSFC under Grant 61371159. Jiming Chen's work is supported by NSFC under Grant 61190110, and National Program for Special Support of Top-Notch Young Professionals. Guoqiang Mao's research is supported by Australian Research Council (AR-C) Discovery projects DP110100538 and DP120102030 and Chinese National Science Foundation project 61428102 .

\section{REFERENCES}

[1] Y. Zhang, S. He, and J. Chen, "Data gathering optimization by dynamic sensing and routing in rechargeable sensor networks," IEEE/ACM Transactions on Networking, DOI: 10.1109/TNET.2015.2425146.

[2] H. Zhang, P. Cheng, L. Shi, and J. Chen, "Optimal dos attack scheduling in wireless networked control system," IEEE Transactions on Control Systems Technology, DOI:10.1109/TCST.2015.2462741.

[3] — "Optimal Denial-of-Service Attack Scheduling with Energy Constraint," IEEE Transactions on Automatic Control, vol. 60, no. 11, pp. 3023-3028, 2015

[4] M. Ding, P. Wang, D. Lopez-Perez, G. Mao, and Z. Lin, "Performance impact of LoS and NLoS transmissions in small cell networks," arXiv preprint arXiv: 1503.04251, 2015.

[5] İ. Güvenç and C.-C. Chong, "A survey on TOA based wireless localization and NLOS mitigation techniques," Communications Surveys \& Tutorials, IEEE, vol. 11, no. 3, pp. 107-124, 2009.

[6] E. U. T. R. Access, "Further advancements for E-UTRA physical layer aspects (release 9), 3GPP," TS, vol. 36, p. V9.

[7] A. SpatialChannel Model, "Spatial channel model text description," 3GPP-3GPP2, Tech. Rep, 2003.

[8] J. Borras, P. Hatrack, and N. B. Mandayam, "Decision theoretic framework for NLOS identification," in 48th IEEE Vehicular Technology Conference (VTC), 1998.

[9] L. Cong and W. Zhuang, "Nonline-of-sight error mitigation in mobile location," IEEE Transactions on Wireless Communications, vol. 4, no. 2 , pp. 560-573, 2005.

[10] Z. Xiao, H. Wen, A. Markham, N. Trigoni, P. Blunsom, and J. Frolik, "Non-line-of-sight identification and mitigation using received signal strength," IEEE Transactions on Wireless Communications, vol. 14, no. 3, pp. 1689-1702, 2015.

[11] X. Shi, Y. H. Chew, C. Yuen, and Z. Yang, "A rss-ekf localization method using hmm-based los/nlos channel identification," in IEEE International Conference onCommunications (ICC), 2014.

[12] P.-C. Chen, "A non-line-of-sight error mitigation algorithm in location estimation," in IEEE Wireless Communications and Networking Conference (WCNC), 1999.

[13] T. V. Nguyen, Y. Jeong, H. Shin, and M. Z. Win, "Least square cooperative localization," IEEE Transactions on Vehicular Technology, vol. 64, no. 4, pp. 1318-1330, 2015.

[14] K. W.-K. Lui, H. C. So, and W.-K. Ma, "Maximum a posteriori approach to time-of-arrival-based localization in non-line-of-sight environment," IEEE Transactions on Vehicular Technology, vol. 59, no. 3, pp. 1517 1523, 2010.

[15] B. Denis and N. Daniele, "Nlos ranging error mitigation in a distributed positioning algorithm for indoor UWB ad-hoc networks," in IEEE International Workshop on Wireless Ad-Hoc Networks, 2004.

[16] K. Yu and Y. J. Guo, "Improved positioning algorithms for nonlineof-sight environments," IEEE Transactions on Vehicular Technology, vol. 57, no. 4, pp. 2342-2353, 2008.

[17] S. Venkatesh and R. M. Buehrer, "NLOS mitigation using linear programming in ultrawideband location-aware networks," IEEE Transactions on Vehicular Technology, vol. 56, no. 5, pp. 3182-3198, 2007.

[18] C. Galiotto, N. K. Pratas, N. Marchetti, and L. Doyle, "A stochastic geometry framework for LOS/NLOS propagation in dense small cell networks," arXiv preprint arXiv:1412.5065, 2014.

[19] Y. Qi, H. Kobayashi, and H. Suda, "Analysis of wireless geolocation in a non-line-of-sight environment," IEEE Transactions on Wireless Communications, vol. 5, no. 3, pp. 672-681, 2006.

[20] S. M. Kay, "Fundamentals of statistical signal processing: estimation theory," 1993.

[21] F. W. Olver, NIST handbook of mathematical functions. Cambridge University Press, 2010.

[22] "http://mathworld.wolfram.com/hermite-gaussquadrature.html."

[23] D. Dardari and F. Sottile, "WPR. B database: Annex of N++ deliverable WPR. B DB. 3. progress report II on advanced localization and positioning techniques: Data fusion and applications," Deliverable Number: $D B$, vol. 3, 2010 . 